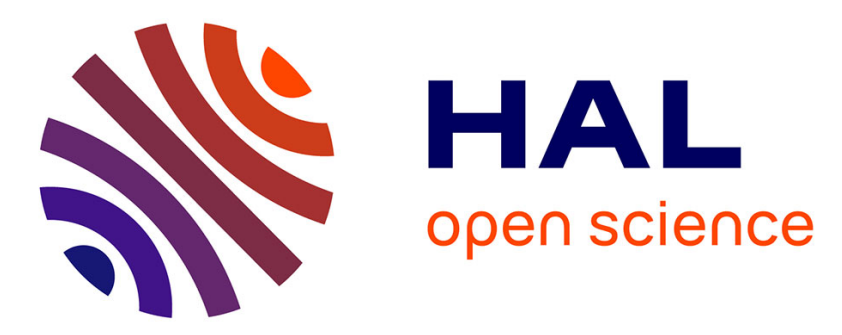

\title{
Retrieval of the polarization ellipse of a THz-wave from precision measurements of lightshifts with cold trapped $\mathrm{HD}+$
}

\author{
Florin Lucian Constantin
}

\section{- To cite this version:}

Florin Lucian Constantin. Retrieval of the polarization ellipse of a THz-wave from precision measurements of lightshifts with cold trapped HD+. SPIE Future Sensing Technologies, Nov 2020, Tokyo (on line), Japan. pp.115251D, 10.1117/12.2581874 . hal-03431790

\author{
HAL Id: hal-03431790 \\ https://hal.science/hal-03431790
}

Submitted on 16 Nov 2021

HAL is a multi-disciplinary open access archive for the deposit and dissemination of scientific research documents, whether they are published or not. The documents may come from teaching and research institutions in France or abroad, or from public or private research centers.
L'archive ouverte pluridisciplinaire HAL, est destinée au dépôt et à la diffusion de documents scientifiques de niveau recherche, publiés ou non, émanant des établissements d'enseignement et de recherche français ou étrangers, des laboratoires publics ou privés.

$$
\text { Copyright }
$$




\title{
Retrieval of the polarization ellipse of a $\mathrm{THz}$-wave from precision measurements of lighshifts with cold trapped $\mathrm{HD}^{+}$
}

\author{
F. L. Constantin ${ }^{*}$
}

Laboratoire PhLAM, CNRS UMR 8523, 59655 Villeneuve d'Ascq, France

\begin{abstract}
THz-waves can be characterized with SI-traceability from the comparison between precision measurements with cold trapped $\mathrm{HD}^{+}$ions and accurate predictions of the molecular ion theory. A THz-wave off-resonantly coupled to the rotational transition at $1.3 \mathrm{THz}$ can be detected via the measurement of the lightshift induced on a Zeeman component of the two-photon rovibrational transition at $55.9 \mathrm{THz}$. A set of six lightshift measurements for two orientations of the magnetic field in the ion trap can be converted into the amplitudes and the phases of the THz electric field components in the orthogonal laboratory frame.
\end{abstract}

Keywords: electrometer, $\mathrm{THz}$ sensing, two-photon spectroscopy, trapped molecular ions, dynamic Stark shift, Zeeman effect, calibration, fundamental constants

*fl.constantin@univ-lille1.fr 


\section{INTRODUCTION}

Atom-based measurements were exploited for frequency standards, inertial force sensing, magnetometry, and testing the fundamental symmetries. The research in electric field sensing was focused recently on Rydberg atoms ${ }^{1}$. The microwave electric field measurements based on Rydberg atom spectroscopy allowed sub-wavelength resolution, accuracy, long-term reproducible operation and SI-traceability. Scalar detection of microwave electric fields at the $\mu \mathrm{V} / \mathrm{cm}$ level was performed with a sensitivity limited by the photon shot noise ${ }^{2}$.

This contribution proposes an extension of this approach to the $\mathrm{THz}$ electric fields by using precision vibrational spectroscopy with cold trapped $\mathrm{HD}^{+}$ions. The energy levels ${ }^{3,4}$ of the hydrogen molecular ions (HMI), and their shifts in external fields ${ }^{5,6,7}$ were predicted accurately by ab-initio calculations. Infrared spectroscopy of trapped and sympathetically cooled HMI provided accurate results $8,9,10,11$. The sub-Doppler spectroscopy techniques, allowing significant improvements of the resolution and of the accuracy, were addressed recently. The fundamental rotational transition of $\mathrm{HD}^{+}$was measured $^{12}$ with a fractional resolution of $3 \times 10^{-12}$ and a fractional uncertainty of $1.3 \times 10^{-11}$. A twophoton rovibrational transition was probed ${ }^{13}$ with a precision of $2.9 \times 10^{-12}$. The sensitivity of the $\mathrm{HD}^{+}$molecular ion transitions to external fields may be exploited to detect the electromagnetic fields and to calibrate them, by comparing the measurements of the systematic effects with the predictions provided by the molecular ion theory. This contribution proposes to characterize a THz-wave offresonantly coupled to the fundamental rotational transition at $1.3 \mathrm{THz}$ by measurements of the lightshifts induced on a Zeeman component of the two-photon rovibrational transition at $55.9 \mathrm{THz}$. Mapping an electromagnetic wave in three dimensions is a nontrivial approach. An algorithm is proposed to convert a set of scalar phase-less lightshift measurements performed for two orientations of the magnetic field in the ion trap into the amplitudes and the phases of the $\mathrm{THz}$ electric field components in the Cartesian laboratory frame. 


\section{MATERIAL AND METHODS}

\subsection{Energy levels and shifts in external fields of $\mathbf{H D}^{+}$}

The rovibrational energy levels of $\mathrm{HD}^{+}$in its ground electronic state were predicted without taking into account the hyperfine structure by ab-initio calculations ${ }^{14}$. The hyperfine structure of the energy levels is described with the coupling of the proton $\vec{I}_{p}$, deuteron $\vec{I}_{d}$, and electron $\vec{S}_{e}$ spins with the rotational angular momentum $\overrightarrow{\mathrm{L}}$ that yields the total angular momentum $\overrightarrow{\mathrm{J}}$ :

$\overrightarrow{\mathrm{F}}=\overrightarrow{\mathrm{S}}_{\mathrm{e}}+\overrightarrow{\mathrm{I}}_{\mathrm{p}} ; \overrightarrow{\mathrm{S}}=\overrightarrow{\mathrm{F}}+\overrightarrow{\mathrm{I}}_{\mathrm{d}} ; \overrightarrow{\mathrm{J}}=\overrightarrow{\mathrm{L}}+\overrightarrow{\mathrm{S}}$. The hyperfine energy levels, labeled with the quantum numbers for the vibration and the spin coupling scheme, were calculated as the eigenvalues of a Breit-type nonrelativistic spin Hamiltonian ${ }^{15}$. The hyperfine states are split in magnetic subcomponents, labelled with the quantum number $\mathrm{J}_{z}$ of the projection of $\vec{J}$ on the quantization axis, by using a static magnetic field. The Zeeman energy levels, calculated as the eigenvalues of a nonrelativistic Hamiltonian $^{5}$, are approximated with a quadratic dependence in function of the strength of the magnetic field, described with Zeeman shift parameters. The total energy of a Zeeman energy level is expressed as :

$$
E\left(v, L, F, S, J, J_{Z}\right)=E_{r v}(v, L)+E_{h f}(v, L, F, S, J)+\Delta E_{Z}(v, L, F, S, J, J Z ; B)
$$

in function of the rovibrational energy $E_{r v}(v, L)$, the hyperfine energy $E_{h f}(v, L, F, S, J)$, and the Zeeman shift $\Delta E_{Z}\left(v, L, F, S, J, J_{Z} ; B\right)$, respectively.

The external fields applied to the ions are described in the Cartesian Laboratory Coordinate Frame $\operatorname{LCF}\left(\vec{e}_{x}, \vec{e}_{y}, \vec{e}_{z}\right)$ shown in Fig. 1.a. The axes of the LCF are oriented along three orthogonal directions defined with the alignment of three coil pairs in Helmholtz configuration, that are exploited to generate a uniform static magnetic field in the ion trap. The electric field of a THz-wave in the $L C F$ is expressed as the sum of three orthogonal linearly polarized components:

$$
\overrightarrow{\mathrm{E}}(\mathrm{t})=\sum_{j=\{x, y, z\}} \frac{E_{j} \vec{e}_{j}}{2} \times \mathrm{e}^{-i\left(\omega t+\varphi_{j}\right)}+\text { c.c. }
$$


in function of three real positive amplitudes $E_{j}$ and two phases $\varphi_{x}, \varphi_{y}$, the third phase is assumed as reference and taken equal to zero $\varphi_{z}=0$.

In addition a Cartesian Molecular Ion Coordinate Frame $\operatorname{MICF}\left(\vec{e}_{c, x}, \vec{e}_{c, y}, \vec{e}_{c, z}\right)$ is defined with the z-axis oriented along the direction of the magnetic field. The $\mathrm{THz}$ electric field is expressed using the standard components in the MICF:

$$
\overrightarrow{\mathrm{E}}(\mathrm{t})=\sum_{q=\{-1,0,1\}}(-1)^{q} \frac{E_{-q} \vec{e}_{q}}{2} \times \mathrm{e}^{-i\left(\omega t+\varphi_{q}\right)}+\text { c.c. }
$$

in function of three real positive amplitudes $E_{0}, E_{ \pm 1}$ and phases $\varphi_{0}, \varphi_{ \pm 1}$. They are associated to the linear or circular polarization components of the THz-wave, characterized with the unit vectors

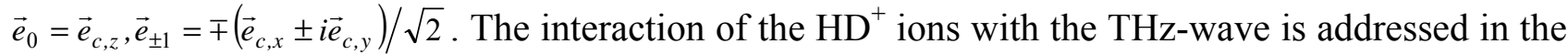
electric dipole approximation with an Hamiltonian:

$$
\mathrm{V}=-\vec{d} \cdot \overrightarrow{\mathrm{E}}(\mathrm{t})
$$

expressed in function of the electric dipole operator $\vec{d}$ of $\mathrm{HD}^{+}$in the laboratory frame.

The lightshift of an energy level of $\mathrm{HD}^{+}$induced by interaction with a THz electric field far offresonance is expressed using the second-order perturbation theory ${ }^{16}$ as:

$$
\begin{gathered}
\delta E_{n}=-\frac{1}{4} \sum_{q=\{-1,0,1\}}(-1)^{q}\left|E_{-q}\right|^{2} \alpha_{n, q}(\omega) \\
\alpha_{n, q}(\omega)=\operatorname{Re}\left[\left\langle n\left|d_{-q} \cdot \frac{1}{\underline{\mathrm{H}}^{*}-\underline{\mathrm{E}}_{n}-\hbar \omega} \cdot d_{q}\right| n\right\rangle+\left\langle n\left|d_{-q} \cdot \frac{1}{\underline{\mathrm{H}}-\underline{\mathrm{E}}_{n}^{*}+\hbar \omega} \cdot d_{q}\right| n\right\rangle\right]
\end{gathered}
$$

The lightshift is calculated in this contribution in function of the dynamic polarizabilities of $\mathrm{HD}^{+}$, that depend on the matrix elements of the standard components of the dipole operator $d_{-q}=\vec{d} \cdot \hat{\varepsilon}_{q}$, the unperturbed energy levels $E_{n}$, and their decay rates $\gamma_{n}$. The second line of eq. (5) introduces the standard dynamic polarizability of $\mathrm{HD}^{+}$in function of the complex operator $\underline{\mathrm{H}}=H+i \hbar \Gamma$, defined such as $\left\langle n|H| n^{\prime}\right\rangle=\delta_{n n^{\prime}} E_{n},\left\langle n|\Gamma| n^{\prime}\right\rangle=\delta_{n n^{\prime}} \gamma_{n} / 2$, and its eigenvalues are $\underline{E}_{n}=E_{n}+i \hbar \gamma_{n} / 2$. 
The standard dynamic polarizability of a $\mathrm{HD}^{+}$energy level $\alpha_{n}\left(\left\{U_{t h}\right\} ; q, B, f_{T H z}\right)$ is calculated in function of a set of theoretical parameters $\left\{U_{t h}\right\}$ (the rovibrational energy levels ${ }^{14}$ with an assumed $10^{-12}$ fractional uncertainty, the hyperfine splittings ${ }^{15}$ with $0.5 \mathrm{kHz}$ uncertainty, the Zeeman shift parameters $^{5}$ with $10^{-4}$ or better fractional uncertainty, the radiative lifetimes of the energy levels ${ }^{17}$ with ms level uncertainty, and the electric dipole transition moments ${ }^{18}$ with $10^{-4}$ fractional uncertainty or better), and a set of experimental parameters (the polarization $q$ of the $\mathrm{THz}$ electric field, the frequency $f_{\mathrm{THz}}$ of the THz-wave with a fractional uncertainty assumed at the ppt level, and the magnetic field strength $B$ with an uncertainty at the $10^{-10} \mathrm{~T}$ level calculated as it is shown in section 2.3). In addition, the uncertainties of the polarizabilities are derived with the error propagation law, using the uncertainties of the theoretical and experimental parameters, and assuming correlations between theoretical parameters of the same type with correlation coefficients equal to 1 .

\subsection{Two-photon spectroscopy scheme of $\mathbf{H D}^{+}$}

This contribution proposes a spectroscopy scheme with cold trapped $\mathrm{HD}^{+}$ions for $\mathrm{THz}$-wave characterization (Fig. 1.a, ${ }^{\mathrm{a}} 1 . \mathrm{b}$ ). The experiment setup is based $\mathrm{b}_{\mathrm{i}}$ an ion trap that stores $\sim 10^{2} \mathrm{HD}^{+}$ ions and $\sim 10^{3}$ laser-cooled $\mathrm{Be}^{+}$ions using a transition at $313 \mathrm{~nm}$. The electrostatic interactions allow sympathetical cooling of the $\mathrm{HD}^{+}$ions, and embed both ions in a Coulomb crystal. The number of $\mathrm{HD}^{+}$ions is determined from the increase of the $313 \mathrm{~nm}$ fluorescence level of $\mathrm{Be}^{+}$ions when the secular motion of the $\mathrm{HD}^{+}$ions is excited in the trap. An uniform static magnetic field, controlled with three coil pairs in the Helmholtz configuration which are driven with three independent current sources, is applied to the ion trap.

First, the two-photon rovibrational transition $(\mathrm{v}, \mathrm{L})=(0,0) \rightarrow(2,0)$ is measured by resonance-enhanced multiphoton dissociation (REMPD) with a stationary laser wave tuned by $55.909 \mathrm{THz}$. The detection is performed by photodissociation of the $(\mathrm{v}, \mathrm{L})=(2,0)$ level with a $175 \mathrm{~nm}$ laser. The time dependences of the populations in the relevant energy levels of the $\mathrm{HD}^{+}$are described by a set of rate equations that take into account the two-photon transition, the dissociation, the radiative relaxation, and the transitions driven by the blackbody radiation. The lineshape of the two-photon resonance is calculated by solving numerically the rate equations ${ }^{19}$. The full-width half measured linewidth of the two-photon rovibrational line is $20 \mathrm{~Hz}$, by assuming a two-photon rovibrational transition rate of 10 
$\mathrm{s}^{-1}$, a dissociation rate of $200 \mathrm{~s}^{-1}$ and a REMPD time of $10 \mathrm{~s}$, that corresponds a fractional resolution beyond the $10^{-12}$ level. The detection time of one experimental point is assumed by $30 \mathrm{~s}$, and the measurement of a $\mathrm{HD}^{+}$line may be performed in about ten minutes. A THz electric field is coupled then off-resonantly to the rotational transition $(\mathrm{v}, \mathrm{L})=(0,0) \rightarrow(0,1)$ at $1.315 \mathrm{THz}$ or $(\mathrm{v}, \mathrm{L})=(2,0) \rightarrow(2,1)$ at $1.197 \mathrm{THz}$, respectively, and another measurement of the two-photon transition allows to derive the lightshift.

The fractional frequency uncertainty of the measurements is estimated with the value of the Allan variance in the case of the molecular ion quantum projection noise limit, expressed as:

$$
\sigma_{y}(\tau)=\frac{1}{\pi Q \sqrt{N_{\text {ion }}}} \times \sqrt{\frac{T_{c}}{\tau}}
$$

in function of the quality factor $Q$ of the two-photon transition, the number $N_{\text {ion }}$ of $\mathrm{HD}^{+}$ions, the cycle time $T_{c}$, and the interrogation time $\tau$. The quality factor $Q=f_{2 p h} / \Delta f_{H W H M}$, is expressed in terms of the two-photon frequency and the half-linewidth, estimated using the natural lifetimes of the $\mathrm{HD}^{+}$ ion energy levels ${ }^{17}$. Assuming the case of single ion spectroscopy experiment with $T_{c}=\tau$, the frequency uncertainty for $(\mathrm{v}, \mathrm{L})=(0,0) \rightarrow(2,0)$ line is estimated at $2.49 \mathrm{~Hz}$ and for $(\mathrm{v}, \mathrm{L})=(0,0) \rightarrow(2,2)$ line at $2.57 \mathrm{~Hz}$.

\subsection{Probing magnetic fields by Zeeman spectroscopy of $\mathbf{H D}^{+}$}

The magnetic field in the ion trap may be also characterized using precision measurements with $\mathrm{HD}^{+}$ ions. This contribution proposes to perform Zeeman spectroscopy of a sensitive component of the two-photon rovibrational transition $(\mathrm{v}, \mathrm{L})=(0,0) \rightarrow(2,2)$ of $\mathrm{HD}^{+}$using a stationary laser wave tuned by 57.701 THz. The detection is performed by photodissociation of the $(\mathrm{v}, \mathrm{L})=(2,2)$ level with the 175 $\mathrm{nm}$ laser. The Zeeman shift of a transition $\left(\mathrm{v}, \mathrm{L}, \mathrm{F}, \mathrm{S}, \mathrm{J}_{\mathrm{Z}} \mathrm{J}_{\mathrm{Z}}\right)=\left(n, J_{z}\right) \rightarrow\left(n^{\prime}, J^{\prime}{ }_{z}\right)$ may be calculated approximately with a quadratic dependence:

$$
f_{\left(n, J_{z}\right) \rightarrow\left(n^{\prime}, J_{z}^{\prime}\right)}(\mathrm{B})-f_{\left(n, J_{z}\right) \rightarrow\left(n^{\prime}, J_{z}^{\prime}\right)}(\mathrm{B}=0)=\eta_{B}\left(\left\{\bigvee_{t h}\right\}, J_{z}, J_{z}^{\prime}\right) B+\eta_{B^{2}}\left(\left\{V_{t h}\right\}, J_{z}, J_{z}^{\prime}\right) B^{2}
$$

in function of the magnitude of the magnetic field $B$ and the linear $\eta_{B}$ and quadratic $\eta_{B^{2}}$ Zeeman shift coefficients that depend on a set of six theoretical parameters $\left\{V_{t h}\right\}=\left\{t_{n}, q_{n}, r_{n}, t_{n^{\prime}}, q_{n^{\prime}}, r_{n^{\prime}}\right\}$, determined for the relevant hyperfine energy levels of $\mathrm{HD}^{+}$in ref. ${ }^{5}$. Solving the second order equation allows to derive the magnitude of the magnetic field in function of the experimental 
Zeeman shift, and the values for the theoretical parameters $\left\{U_{t h}\right\}$. The value of the magnetic field may be derived by inverting the second-order equation and choosing, if necessary, the suitable solution that matches the value of the magnetic field provided, for example, by an accurate magnetometer. The uncertainty of the magnetic field may be calculated with the error propagation formula, in function of the contribution from the uncertainty of the Zeeman shift measurement $u\left(\delta f_{z}\right)=2.57 \cdot \sqrt{2} \mathrm{~Hz}$, estimated with the molecular ion quantum projection noise limit, and from the Zeeman shift parameters uncertainties $u(q)=u(r)=50 \mathrm{MHz} / \mathrm{T}^{2}, u(t)=5 \mathrm{kHz} / \mathrm{T}$.

The dependence of the fractional uncertainty of the magnetic field in function of the magnetic field is calculated and plotted in Fig. 2 for a selection of sensitive transitions starting from each hyperfine level of the $(\mathrm{v}, \mathrm{L})=(0,0)$ state. The transition $\left(\mathrm{v}, \mathrm{L}, \mathrm{F}, \mathrm{S}, \mathrm{J}, \mathrm{J}_{\mathrm{Z}}\right)=(0,0,1,2,2,-2) \rightarrow(2,2,1,2,4,0)$ is the most sensitive and displays the lowest uncertainties. The total uncertainty, by $4 \times 10^{-10} \mathrm{~T}$, indicates the precision of the calibration of the magnetic field by Zeeman spectroscopy. This uncertainty is due mostly to frequency measurement error. The contribution of the theoretical error, shown also in Fig. 2 , is orders of magnitude smaller and remains at a fractional value by $7 \times 10^{-7}$.

\section{RESULTS AND DISCUSSION}

\subsection{Scalar THz electrometry using $\mathrm{HD}^{+}$spectroscopy : sensitivity and precision issues}

The total lightshift induced by a THz-wave on a two-photon rovibrational transition $\left(\mathrm{v}, \mathrm{L}, \mathrm{F}, \mathrm{S}, \mathrm{J}_{\mathrm{Z}} \mathrm{J}_{\mathrm{Z}}\right)=(\mathrm{n}) \rightarrow\left(\mathrm{n}^{\prime}\right)$ may be expressed in function of the squared amplitude of the electric field $E_{T H z}$ of the THz-wave:

$$
f_{n \rightarrow n^{\prime}}\left(E_{T H z}\right)-f_{n \rightarrow n^{\prime}}\left(E_{T H z}=0\right)=-\frac{\left|E_{T H z}\right|^{2}}{8}\left(\alpha_{n^{\prime}}\left(\left\{U_{t h}\right\} ; B, f_{T H z}\right)-\alpha_{n}\left(\left\{U_{t h}\right\} ; B, f_{T H z}\right)\right)
$$

$\mathrm{A} \mathrm{HD}^{+}$frequency measurement may be converted into a measurement of the THz-wave electric field by using the total dynamic polarizability of the $\mathrm{HD}^{+}$energy levels that were calculated theoretically. In the case of the detection of a progressive plane wave, which is assumed here, the $\mathrm{THz}$ electric field can be related to the THz-wave intensity as $I_{T H z}=c \varepsilon_{0} E_{T H z}^{2}$, in function of the speed of the light $\mathrm{c}$, and the vacuum permittivity $\varepsilon_{0}$. 
The uncertainty of the THz-wave intensity is determined by the frequency measurement errors, which translate into the uncertainties of the lightshift and of the magnetic field magnitude, and by the uncertainties of the theoretical parameters, that contribute to the uncertainty of the dynamic polarizability and of the magnetic field magnitude. The uncertainty of a THz-wave intensity measurement is estimated with the error propagation law :

$$
u\left(I_{T H z}\right)=\sqrt{\left(\frac{\left(8 c \varepsilon_{0}\right) \times u\left(\delta f_{L S}\right)}{\Delta \alpha\left(\left\{U_{t h}\right\} ; B, f_{T H z}\right)}\right)^{2}+\left(\frac{I_{T H z} u\left(\Delta \alpha\left(\left\{U_{t h}\right\} ; B, f_{T H z}\right)\right)}{\Delta \alpha\left(\left\{U_{t h}\right\} ; B, f_{T H z}\right)}\right)^{2}}
$$

in function of the uncertainty of the lightshift measurement $u\left(\delta f_{L S}\right)$, and the uncertainty of the dynamic polarizability calculation $u\left(\Delta \alpha\left(\left\{U_{t h}\right\} ; B, f_{T H z}\right)\right)$. The first term on the right side of the eq. (9) gives the sensitivity limit for the detection of the THz-wave by lightshift measurement. In order to minimize the uncertainty calculated with eq. (9), for a given intensity, polarization and frequency of the THz-wave, the two-photon transition should have the highest dynamic polarizability, the lightshift measurements should be performed with the lowest uncertainties, and the dynamic polarizability should be estimated with the lowest uncertainty. Particularly, the value of the magnetic field may be suitably chosen in order to minimize the uncertainty of the THz-wave intensity.

In the case of the detection of a THz-wave at $1,314,947,502.3 \mathrm{kHz}$ with $\pi$ polarization by using lightshift measurements on the $\left(\mathrm{v}, \mathrm{L}, \mathrm{F}, \mathrm{S}, \mathrm{J}, \mathrm{J}_{\mathrm{Z}}\right)=(0,0,1,2,2,2) \rightarrow(2,0,1,2,2,2)$ two-photon transition with an experimental uncertainty of $u\left(\delta f_{L S}\right)=2.49 \cdot \sqrt{2} \mathrm{~Hz}$, the optimization procedure for the magnetic field magnitude yields the smallest experimental uncertainty of the THz-wave intensity of $4.8 \times 10^{-8}$ $\mathrm{W} / \mathrm{m}^{2}$. The limit of sensitivity of this method is a $6 \times 10^{-6} \mathrm{~V} / \mathrm{m} \mathrm{THz}$ electric field, which is a 30 times improvement to the value of the lowest microwave electric field detected previously with the Rydberg atom spectroscopy method ${ }^{2}$.

The uncertainty for the calibration of the intensity of a THz-wave at $1,314,947,502.3 \mathrm{kHz}$ with $\sigma^{-}$or $\pi$ polarization, using lightshift measurements of the $\left(\mathrm{v}, \mathrm{L}, \mathrm{F}, \mathrm{S}, \mathrm{J}, \mathrm{J}_{\mathrm{Z}}\right)=(0,0,1,2,2,2) \rightarrow(2,0,1,2,2,2)$ twophoton transition of $\mathrm{HD}^{+}$, is calculated for a value of the magnetic field up to $2 \times 10^{-4} \mathrm{~T}$, that minimizes the total uncertainty estimated with eq. (9). The results are plotted in Fig. 3. The contribution to the fractional uncertainty arising solely from the frequency measurement errors is by an order of magnitude smaller than the total fractional uncertainty. The precision of the calibration a 
THz-wave with an intensity of $1 \mathrm{~W} / \mathrm{m}^{2}$ is at the $10^{-3}$ level, being limited by the accuracy of the dynamic polarizability calculation.

\subsection{Three-dimensional characterization of a $\mathbf{T H z}$-wave using $\mathrm{HD}^{+}$spectroscopy}

A set of lightshifts induced on the components of the two-photon rovibrational transition $(\mathrm{v}, \mathrm{L})=(0,0) \rightarrow(2,0)$ can be exploited to characterize the polarization ellipse of a THz-wave in three dimensions. As indicated in eq. (5), each standard component of the $\mathrm{THz}$ electric field in the MICF probes off-resonantly $\pi$ or $\sigma^{ \pm}$Zeeman subcomponents of the $(\mathrm{v}, \mathrm{L})=(\mathrm{v}, 0) \rightarrow(\mathrm{v}, 1)$ transitions, and induces a lightshift in proportion with its squared amplitude. In addition, the squared amplitudes $\left|E_{\pi, \sigma^{ \pm}}^{(\alpha, \beta)}\right|^{2}$ of the standard components of the THz electric field can be related in function of the Euler angles $\alpha$ and $\beta$ to the amplitudes and phases of the Cartesian components of the electric field in the $\operatorname{LCF}\left(E_{x}, E_{y}, E_{z}, \varphi_{x}, \varphi_{y}\right)^{20}$. The characterization of the five THz-wave parameters requires at least five independent measurements of the squared amplitudes of the standard components.

This contribution proposes to exploit six independent measurements of lightshifts of two-photon transitions $F_{L S}=\left\{\delta f_{1}^{\left(\alpha_{1}, \beta_{1}\right)}, \delta f_{2}^{\left(\alpha_{1}, \beta_{1}\right)}, \delta f_{3}^{\left(\alpha_{1}, \beta_{1}\right)}, \delta f_{4}^{\left(\alpha_{2}, \beta_{2}\right)}, \delta f_{5}^{\left(\alpha_{2}, \beta_{2}\right)}, \delta f_{6}^{\left(\alpha_{2}, \beta_{2}\right)}\right\}$ for two different orientations of the magnetic field. The lightshifts are related to the squared amplitudes of the standard components of the THz electric field $E_{\text {STD }}^{2}=\left\{\left|E_{\sigma^{+}}^{\left(\alpha_{1}, \beta_{1}\right)}\right|^{2},\left|E_{\pi}^{\left(\alpha_{1}, \beta_{1}\right)}\right|^{2},\left|E_{\sigma^{-}}^{\left(\alpha_{1}, \beta_{1}\right)}\right|^{2},\left|E_{\sigma^{+}}^{\left(\alpha_{2}, \beta_{2}\right)}\right|^{2},\left|E_{\pi}^{\left(\alpha_{2}, \beta_{2}\right)}\right|^{2},\left|E_{\sigma^{-}}^{\left(\alpha_{2}, \beta_{2}\right)}\right|^{2}\right\}$ for the chosen orientations with a $6 \times 6$ matrix $A\left(\Delta \alpha_{i, q}\left(\left\{U_{t h}\right\} ; B, f_{T H z}\right)\right)$ formed with two $3 \times 3$ diagonal blocks depending on the appropriate differential standard dynamic polarizabilities of the two-photon transitions multiplied with the suitable prefactors. The squared amplitudes of the standard components may be determined by inversion $\mathrm{E}_{\mathrm{STD}}^{2}=\left[\mathrm{A}\left(\Delta \alpha_{\mathrm{i}, \mathrm{q}}\right)\right]^{-1} \cdot \mathrm{F}_{\mathrm{LS}}$, if the matrix is nonsingular. This requirement can be satisfied by using three different values of the magnetic field, or by addressing three different Zeeman or hyperfine subcomponents of the two-photon transition.

The choice of the orientations of the magnetic field should take into account the periodicity of the transformation from the squared amplitudes of the standard components of the $\mathrm{THz}$ electric field in the MICF to the Cartesian components of the THz electric field in the LCF. The two orientations chosen here are described with Euler angles $\left(\alpha_{1}, \beta_{1}\right)=(0,0)$ and $\left(\alpha_{2}, \beta_{2}\right)=(0, \pi / 2)$. In this case, the 
parameters of the THz-wave in the LCF may be expressed with analytical dependences in function of $\left(\left|E_{\pi}^{(0,0)}\right|^{2},\left|E_{\sigma^{+}}^{(0,0)}\right|^{2},\left|E_{\sigma^{-}}^{(0,0)}\right|^{2},\left|E_{\sigma^{+}}^{(0, \pi / 2)}\right|^{2},\left|E_{\sigma^{-}}^{(0, \pi / 2)}\right|^{2}\right):$

$$
\begin{aligned}
& E_{x}=\sqrt{\left|E_{\sigma^{+}}^{(0,0)}\right|^{2}+\left|E_{\sigma^{-}}^{(0,0)}\right|^{2}-\left(\left|E_{\sigma^{+}}^{(0, \pi / 2)}\right|^{2}+\left|E_{\sigma^{-}}^{(0, \pi / 2)}\right|^{2}-\left|E_{\pi}^{(0,0)}\right|^{2}\right)} \\
& E_{y}=\sqrt{\left|E_{\sigma^{+}}^{(0, \pi / 2)}\right|^{2}+\left|E_{\sigma^{-}}^{(0, \pi / 2)}\right|^{2}-\left|E_{\pi}^{(0,0)}\right|^{2}} \\
& E_{z}=\left|E_{\pi}^{(0,0)}\right| \\
& \varphi_{x}=\varphi_{y}+\arcsin \left(\frac{\left|E_{\sigma^{+}}^{(0,0)}\right|^{2}-\left|E_{\sigma^{-}}^{(0,0)}\right|^{2}}{2 E_{x} E_{y}}\right) \\
& \varphi_{y}=\arcsin \left(\frac{\left|E_{\sigma^{+}}^{(0, \pi / 2)}\right|^{2}-\left|E_{\sigma^{-}}^{(0, \pi / 2)}\right|^{2}}{2 E_{y} E_{z}}\right)
\end{aligned}
$$

Let us discuss the application of this method for the characterization of a reference THz-wave that is $\sigma^{+}$polarized and propagates along an arbitrary direction defined in the LCF with Euler angles $\phi$ and $\theta$ (Fig. 1.a). The parameters of the THz-wave in the LCF may be expressed as:

$$
\begin{aligned}
& E_{x}=\left(E_{0} / \sqrt{2}\right) \cdot \sqrt{\cos ^{2}(\theta) \cos ^{2}(\phi)+\sin ^{2}(\phi)} \\
& E_{y}=\left(E_{0} / \sqrt{2}\right) \cdot \sqrt{\cos ^{2}(\theta) \sin ^{2}(\phi)+\cos ^{2}(\phi)} \\
& E_{z}=\left(E_{0} / \sqrt{2}\right) \cdot \sin (\phi) \\
& \varphi_{x}=\left\{\begin{array}{l}
\arccos \left(\frac{-\cos (\theta) \cos (\phi)}{\sqrt{\cos ^{2}(\theta) \cos ^{2}(\phi)+\sin ^{2}(\phi)}}\right) \text { if } \sin (\phi) \geq 0 \\
-\arccos \left(\frac{-\cos (\theta) \cos (\phi)}{\sqrt{\cos ^{2}(\theta) \cos ^{2}(\phi)+\sin ^{2}(\phi)}}\right) \text { if } \sin (\phi)<0
\end{array}\right) \text { if } \cos (\phi) \leq 0 \\
& \arccos \left(\frac{-\cos (\theta) \sin (\phi)}{\left.\sqrt{\cos ^{2}(\theta) \sin ^{2}(\phi)+\cos ^{2}(\phi)}\right)}\right) \text { if } \cos (\phi)>0 \\
& -\arccos \left(\frac{-\cos (\theta) \sin (\phi)}{\sqrt{\cos ^{2}(\theta) \sin ^{2}(\phi)+\cos ^{2}(\phi)}}\right)
\end{aligned}
$$

in function of the amplitude of the THz-wave $\mathrm{E}_{0}$. The frequency of the $\mathrm{THz}$ wave is assumed at $\mathrm{f}_{\mathrm{THz}}$ $=1,314,947,502.3 \mathrm{kHz}$, the intensity at $1 \mathrm{~W} / \mathrm{m}^{2}\left(\mathrm{E}_{0}=27.42 \mathrm{mV} / \mathrm{m}\right)$ and the direction is defined by $(\phi=\pi / 4, \theta=\pi / 4)$. 
The measurements address six lightshifts of the two-photon transition $\left(\mathrm{v}, \mathrm{L}, \mathrm{F}, \mathrm{S}, \mathrm{J}, \mathrm{J}_{\mathrm{z}}\right)=(0,0,1,2,2,2) \rightarrow(2,0,1,2,2,2)$ of $\mathrm{HD}^{+}$for two orientations of the magnetic field $(\alpha, \beta)=(0,0)$ and $(\alpha, \beta)=(0, \pi / 2)$ in the case of three different values of the magnetic field $\mathrm{B}_{1}=10^{-6} \mathrm{~T}, \mathrm{~B}_{2}=$ $5 \times 10^{-6} \mathrm{~T}$, and $\mathrm{B}_{3}=10^{-5} \mathrm{~T}$. First, the experimental lightshifts are simulated by adding the values of the lightshifts calculated for the reference THz-wave and random errors described with a Gaussian distribution with zero mean and covariance equal to the square of the experimental uncertainty $u\left(\delta f_{L S}\right)=2.49 \cdot \sqrt{2} \mathrm{~Hz}$. The squared standard components of the $\mathrm{THz}$ electric field are subsequently derived using the relevant set of differential standard dynamic polarizabilities. The polarizabilities are estimated as the theoretical values plus a random contribution with a Gaussian distribution with zero mean and variance equal to the squared uncertainty of each polarizability calculation. Then, the parameters of the $\mathrm{THz}$ electric field in the laboratory frame are determined analytically from the calculated values of the squared standard components. The retrieved polarization ellipses of the $\mathrm{THz}$ electric field are represented on Fig. 4 for 10 simulated sets of lightshifts. The mean values and the uncertainties of the parameters of the THz-wave are:

$$
\begin{gathered}
E_{x}=16.39(84) \mathrm{mV} / \mathrm{m}, E_{y}=17.11(56) \mathrm{mV} / \mathrm{m}, E_{z}=13.709(24) \mathrm{mV} / \mathrm{m} \\
\varphi_{x}=2.21(20) \mathrm{rad}, \varphi_{y}=-2.24(11) \mathrm{rad}
\end{gathered}
$$

The calibration accuracy for the Cartesian components of the $\mathrm{THz}$ electric field in the laboratory frame is $5 \%$ or better for the amplitudes, and better than $10 \%$ for the phases. For each parameter of the THz-wave, the deviation between the mean value obtained by measurement and the corresponding value of the reference THz-wave is compatible with the uncertainty of the measurement. The comparison of the projections on the $x y, y z$, and zx planes (Fig. 4) of the polarization ellipse of the reference $\mathrm{THz}$-wave and of a polarization ellipse calculated with the parameters from eq. (12) confirms qualitatively the accurate retrieval of the polarization ellipse of the THz-wave by lightshift measurements. 


\section{CONCLUSION}

This contribution proposes a new method to characterize the electromagnetic fields based on the comparison of the measurements of the frequency shifts induced on two-photon rovibrational transitions of cold trapped $\mathrm{HD}^{+}$ions with the predictions of the molecular ion theory. The SItraceability is ensured on one hand, by performing infrared frequency measurements with an assumed fractional uncertainty at the $10^{-12}$ level against a frequency standard and, on the other hand, by exploiting accurate ab-initio calculations of the energy levels and of the transition dipole moments based on the values of the fundamental physical constants ${ }^{21}$. The approach allows to derive the uncertainties of the parameters of the electromagnetic fields in function of the uncertainties of the frequency measurements and of the theoretical calculations.

The Zeeman spectroscopy of the $\left(\mathrm{v}, \mathrm{L}, \mathrm{F}, \mathrm{S}, \mathrm{J}, \mathrm{J}_{\mathrm{Z}}\right)=(0,0,1,2,2,-2) \rightarrow(2,2,1,2,4,0)$ transition of $\mathrm{HD}^{+}$may allow the measurement of the magnitude of a static magnetic field applied to the ion trap with an uncertainty at the $10^{-10} \mathrm{~T}$ level. In addition, the amplitude of the electric field of a THz-wave with linear or circular polarization tuned near the hyperfine transition $(\mathrm{v}, \mathrm{L}, \mathrm{F}, \mathrm{S}, \mathrm{J})=(0,0,1,2,2) \rightarrow(0,1,1,2,3)$ of $\mathrm{HD}^{+}$may be characterized using the lightshift induced on the transition $\left(\mathrm{v}, \mathrm{L}, \mathrm{F}, \mathrm{S}, \mathrm{J}_{\mathrm{Z}} \mathrm{J}_{\mathrm{Z}}\right)=(0,0,1,2,2,2) \rightarrow(2,0,1,2,2,2)$. The sensitivity of the precision spectroscopy-based method is at the $10^{-6} \mathrm{~V} / \mathrm{m}$ level. A THz-wave with an intensity of $1 \mathrm{~W} / \mathrm{m}^{2}$ can be calibrated using lightshift measurements with a precision at the $10^{-3}$ level, determined by the accuracy of the theoretical calculations. In addition, the electric field vector of the THz-wave may be characterized in three dimensions using lightshift measurements for different orientations of the magnetic field in the ion trap. The response of $\mathrm{HD}^{+}$to the external field obtained with this method may be referenced directly to a frequency standard, that is advantageous comparing to the approaches based on the Rabi-rate measurements ${ }^{20,22}$. A THz-wave with circular polarization and an intensity of $1 \mathrm{~W} / \mathrm{m}^{2}$ may be calibrated using a set of ten lightshift measurements with a precision estimated at $5 \%$ or better for the amplitudes of the electric field components in the Cartesian laboratory frame and better than $10 \%$ for the phases. 


\section{REFERENCES}

[1] Sedlacek, J.A. et al, "Microwave electrometry with Rydberg atoms in a vapour cell using bright atomic resonances," Nat. Phys. 8, 819-824 (2012).

[2] Kumar, S. et al, "Rydberg-atom based radio-frequency electrometry using frequency modulation spectroscopy in room temperature vapour cells," Opt. Express 25 (8), 8625-8637 (2017). [3] Korobov, V.I., Hilico, L. and Karr, J.-Ph., "Fundamental transitions and ionization energies of the hydrogen molecular ions with few ppt uncertainty," Phys. Rev. Lett. 118 (23), 233001 (2017).

[4] Korobov, V.I., Karr, J.-Ph., Haidar, M. and Zhong, Z.-X., "Hyperfine structure in the $\mathrm{H}_{2}{ }^{+}$and $\mathrm{HD}^{+}$molecular ions at order ma $\alpha^{6}$, Phys. Rev. A 102 (2), 022804 (2020).

[5] Bakalov, D., Korobov, V.I. and Schiller, S., "Magnetic field effects in the transitions of the $\mathrm{HD}^{+}$molecular ion and precision spectroscopy,” J. Phys. B 44 (2), 025003 (2011).

[6] Schiller, S., Bakalov, D., Bekbaev, A.K. and Korobov, V.I., "Static and dynamic polarizability and the Stark and blackbody-radiation frequency shifts of the molecular hydrogen ions $\mathrm{H}_{2}^{+}, \mathrm{HD}^{+}$, and $\mathrm{D}_{2}^{+}$, , Phys. Rev. A 89 (5), 052521 (2014).

[7] Bakalov, D. and Schiller, S., "The electric quadrupole moment of molecular hydrogen ions and their potential for a molecular ion clock," Appl. Phys. B 114 (1-2), 213-230 (2014).

[8] Leach, C.A. and Moss, R.E., "Spectroscopy and quantum mechanics of the hydrogen molecular cation: A test of molecular quantum mechanics,” Annu. Rev. Phys. Chem. 46, 55-82 (1995).

[9] Koelemeij, J.C.J., Roth, B., Wicht, A., Ernsting, I. and Schiller, S., "Vibrational spectroscopy of $\mathrm{HD}^{+}$with 2-ppb accuracy,” Phys. Rev. Lett. 98 (17), 173002 (2007).

[10] Bressel, U., Borodin, A., Shen, J., Hansen, M., Ernsting, I. and Schiller, S. "Manipulation of individual hyperfine states in cold trapped molecular ions and application to $\mathrm{HD}^{+}$frequency metrology,” Phys. Rev. Lett. 108 (18), 183003 (2012).

[11] Biesheuvel, J., Karr, J.-Ph., Hilico, L., Eikema, K.S.E., Ubachs W. and Koelemeij, J.C.J., "Probing QED and fundamental constants through laser spectroscopy of vibrational transitions in $\mathrm{HD}^{+}$, , Nat. Commun. 7, 10385 (2016). 
[12] Alighanbari, S., Giri, G.S., Constantin, F.L., Korobov, V. and Schiller, S., "Precise test of quantum electrodynamics and determination of fundamental constants with $\mathrm{HD}^{+}$ions," Nature 581, $152-158$ (2020).

[13] Patra, S. et al, "Proton-electron mass ratio from laser spectroscopy of $\mathrm{HD}^{+}$at the part-pertrillion level," Science 369 (6508), 1238-1241 (2020).

[14] Moss, R.E., "Calculations for vibration-rotation levels of $\mathrm{HD}^{+}$, in particular for high N," Mol. Phys. 78 (2), 371-405 (1993).

[15] Bakalov, D., Korobov, V.I. and Schiller, S., "High-precision calculation of the hyperfine structure of the HD ${ }^{+}$ion,” Phys. Rev. Lett. 97 (24), 243001 (2006).

[16] Le Kien, F., Schneeweiss, P. and Rauschenbeutel, A., "Dynamical polarizability of atoms in arbitrary light fields: General theory and application to cesium,” Eur. Phys. J. D 67 (5), 92 (2013).

[17] Amitay, Z., Zajfman, D. and Forck, P., "Rotational and vibrational lifetime of isotopically asymmetrized homonuclear diatomic molecular ions," Phys. Rev. A 50 (3), 2304-2308 (1994).

[18] Bakalov, D. and Schiller S., "Static Stark effect in the molecular ion HD", Hyperfine Interact. $210(1-3), \quad 25-31(2012)$.

[19] Constantin, F.L., "Double-resonance two-photon spectroscopy of hydrogen molecular ions for improved determination of fundamental constants," IEEE Trans. Instrum. Meas. 68 (6), 21512159 (2019).

[20] Koepsell, J., Thiele, T., Deiglmayr, J., Wallraff, A. and Merkt, F., "Measuring the polarization of electromagnetic fields using Rabi-rate measurements with spatial resolution : Experiment and theory," Phys. Rev. A 95 (5), 053860 (2017).

[21] Mohr, P.J., Newell, D.B. and Taylor, B.N., "CODATA recommended values of the fundamental physical constants: 2014,” Rev. Mod. Phys. 88 (3), 035009 (2016).

[22] Thiele, T., Lin, Y., Brown, M.O. and Regal, C.A., "Self-calibrating vector atomic magnetometry through microwave polarization reconstruction,” Phys. Rev. Lett. 121 (15), 153202 (2018). 


\section{Figure captions:}

Figure 1. a. The experimental setup with the coordinate frames. $\mathrm{Be}^{+}$ions are cooled with the $313 \mathrm{~nm}$ laser (magenta line). Two-photon spectroscopy of $\mathrm{HD}^{+}$is performed with a stationary wave from the IR laser (red line). The detection is performed by dissociating $\mathrm{HD}^{+}$ions with the $175 \mathrm{~nm}$ laser (green line). The static magnetic field in the ion trap (olive line) can be oriented to any direction, defined with Euler angles $\alpha$ and $\beta$ in the Cartesian Laboratory Coordinate Frame (x,y,z) (black lines) $(L C F)$. The THz-wave (blue line), off-resonantly coupled to the energy levels of the $\mathrm{HD}^{+}$ions, has a wave vector $\overrightarrow{\mathrm{n}}$ oriented in a direction defined with the Euler angles $\theta$ and $\phi$ in the LCF. The standard components of the $\mathrm{THz}$ electric field are referenced in the Cartesian Molecular Ion Coordinate Frame $\left(\mathrm{x}_{\mathrm{c}}, \mathrm{y}_{\mathrm{c}}, \mathrm{z}_{\mathrm{c}}\right)$ (black lines) $(M I C F)$. The MICF is related to the LCF by a $\mathrm{z}-\mathrm{y}-\mathrm{z}$ rotation with the Euler angles $(\alpha, \beta, \chi=0)$. b. Rotation-vibration energy levels of $\mathrm{HD}^{+}$addressed in the $\mathrm{THz}$ sensing scheme. The two-photon rovibrational transitions are indicated with red lines with arrows, the dissociation with green lines with arrows, the THz-wave-driven electric dipole couplings with blue lines with arrows and the blackbody-radiation-driven transitions with black lines and curves with arrows.
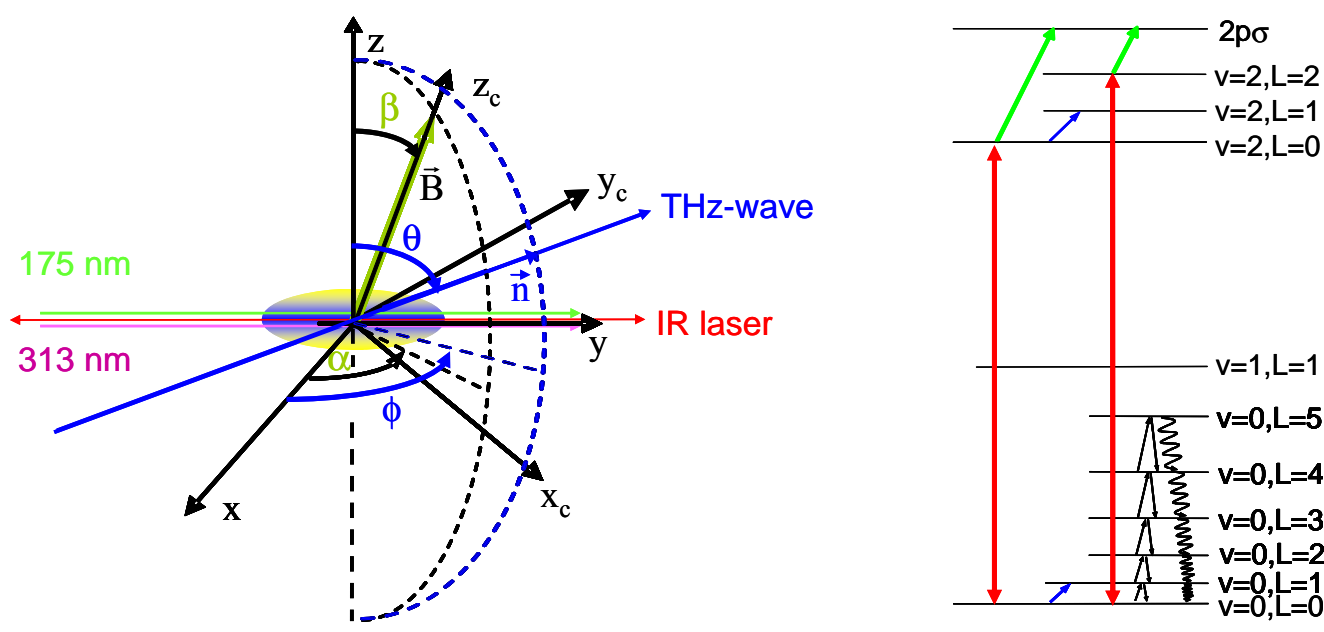
Figure 2. Dependence of the fractional uncertainty of the magnetic field determined by Zeeman spectroscopy in function of the magnitude of the magnetic field for a selection of two-photon transitions. The dependences of the combined experimental and theoretical fractional uncertainties are represented with continuous lines. The dependences of the theoretical fractional uncertainties are represented with dotted lines.

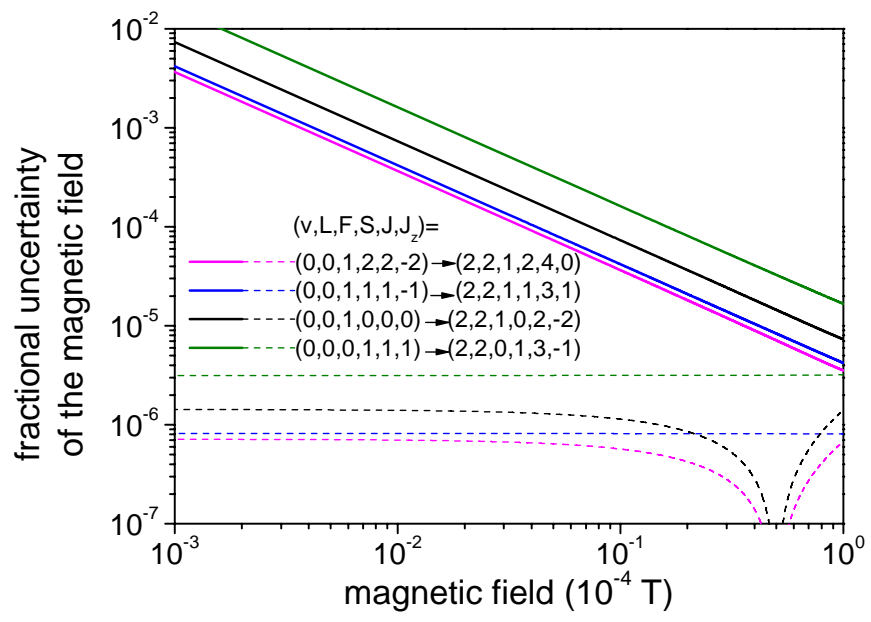

Figure 3. Dependence of the fractional uncertainty of the THz-wave intensity in function of the THz-wave intensity (left axis, bottom axis). THz-wave polarization $\sigma^{-}$(red lines) and $\pi$ (blue lines). The combined experimental and theory errors are shown with continuous lines. The experimental errors are shown with dotted lines. The plot indicates also the dependence of the fractional uncertainty of the $\mathrm{THz}$ electric field amplitude in function of the $\mathrm{THz}$ electric field amplitude (right axis, top axis). 


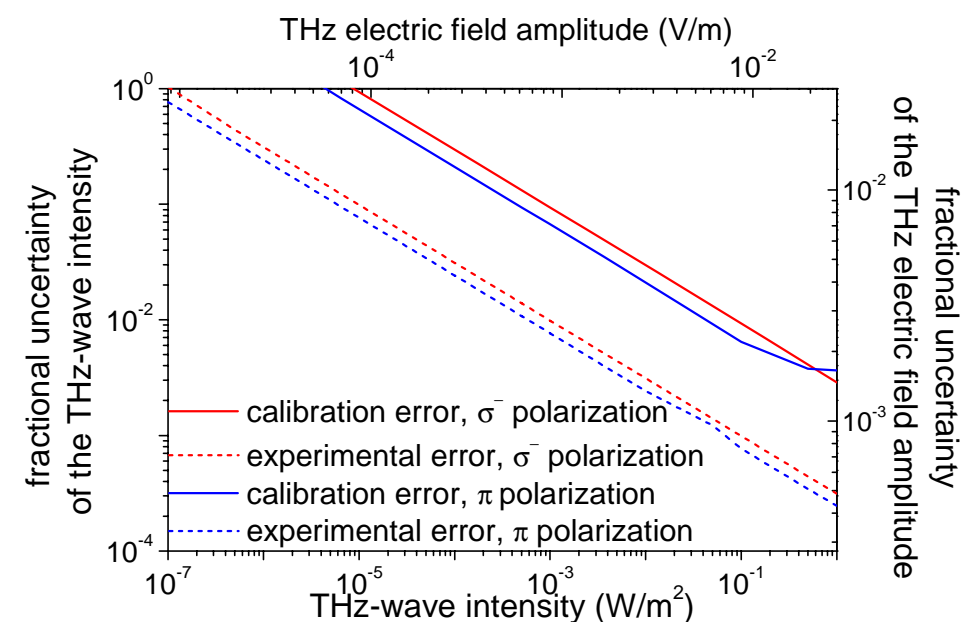


Figure 4. Polarization ellipse of the reference THz-wave (red ellipse) and polarization ellipses retrieved from lightshift measurements (blue ellipses). The projections on the $\mathrm{xy}, \mathrm{yz}$, and $\mathrm{zx}$ planes of the polarization ellipse of the reference THz-wave are shown in orange. The projections on the $\mathrm{xy}, \mathrm{yz}$, and $\mathrm{zx}$ planes of the polarization ellipse calculated with the mean values of the THz-wave parameters obtained by measurements are shown in black.

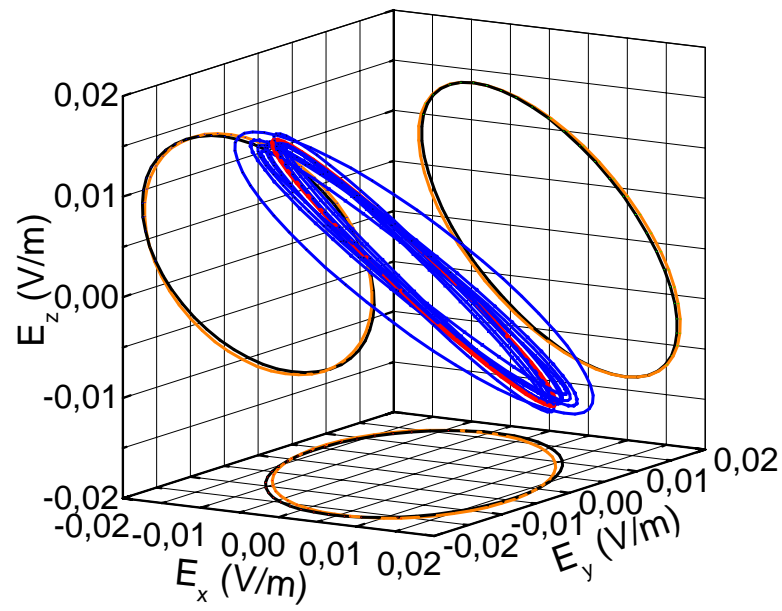

